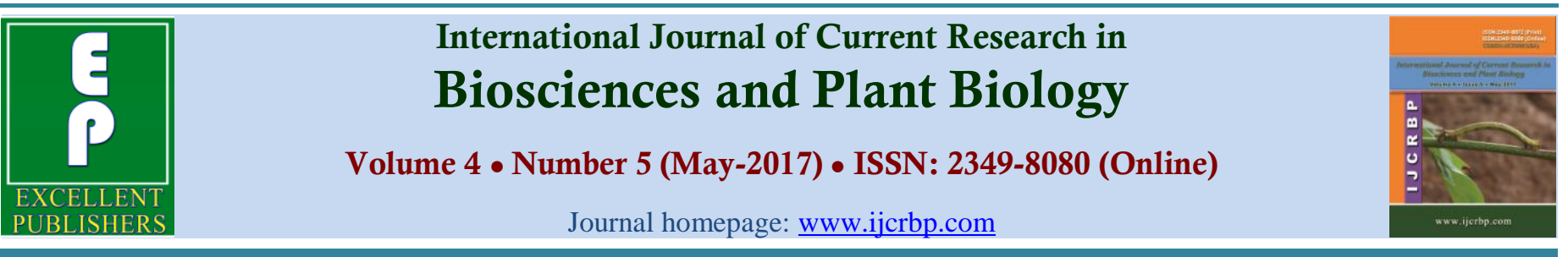

\title{
R Gene and its Role in Disease Managements in Plants
}

\author{
Tarun Kumar Jatwa ${ }^{1}$, Meenakshi Sharma ${ }^{1}$ and Ashok Kumar Malav ${ }^{2}$ \\ ${ }^{I}$ Department of Plant Pathology, Rajasthan College of Agriculture, Maharana Pratap University of Agriculture and Technology, \\ Udaipur-313001, Rajasthan, India \\ ${ }^{2}$ Department of Plant Breeding and Genetics, Rajasthan College of Agriculture, Maharana Pratap University of Agriculture and \\ Technology, Udaipur-313001, Rajasthan, India
}

*Corresponding author.

\begin{tabular}{|c|c|}
\hline Abstract & Article Info \\
\hline \multirow{7}{*}{$\begin{array}{l}\text { Many disease resistance }(\mathrm{R}) \text { proteins of plants detect the presence of disease-causing } \\
\text { bacteria, viruses, or fungi by recognizing specific pathogen effector molecules that are } \\
\text { produced during the infection process. Effectors are often pathogen proteins that probably } \\
\text { evolved to subvert various host processes for promotion of the pathogen life cycle. Five } \\
\text { classes of effector-specific } \mathrm{R} \text { proteins are known, and their sequences suggest roles in both } \\
\text { effector recognition and signal transduction. The most prevalent class of } \mathrm{R} \text { proteins } \\
\text { contain leucine-rich repeats (LRRs), a central nucleotide binding site and a variable amino } \\
\text { terminal domain. Other classes possess an extracellular LRR domain, a transmembrane } \\
\text { domain and sometimes an intracellular serine/threonine kinase domain. } \mathrm{R} \text { proteins } \\
\text { function in pathogen perception and/or the activation of conserved defence signalling } \\
\text { networks. Upon infection, specific effectors produced by pathogens and presumed to } \\
\text { promote growth in host tissue, are either directly recognized by different } \mathrm{R} \text { proteins or are } \\
\text { recognized by a targeted plant protein which is itself guarded by } \mathrm{R} \text { proteins. }\end{array}$} & $\begin{array}{l}\text { Accepted: } 29 \text { April } 2017 \\
\text { Available Online: } 06 \text { May } 2017\end{array}$ \\
\hline & Keywords \\
\hline & Avirulence genes \\
\hline & Disease resistance proteins \\
\hline & Plant pathogens \\
\hline & Resistance gene \\
\hline & \\
\hline
\end{tabular}

\section{Introduction}

Plants have evolved $R$ genes (resistance genes) whose products mediate resistance to specific virus, bacteria, oomycete, fungus, nematode or insect strains. $\mathrm{R}$ gene products are proteins that allow recognition of specific pathogen effectors, either through direct binding or by recognition of the effector's alteration of a host protein. Many $R$ genes encode NB-LRR proteins (proteins with nucleotide-binding and leucine-rich repeat domains, also known as NLR proteins or STAND proteins, among other names). Most plant immune systems carry a repertoire of 100-600 different $\mathrm{R}$ gene homologs. Individual $R$ genes have been demonstrated to mediate resistance to specific virus, bacteria, oomycete, fungus, nematode or insect strains. $\mathrm{R}$ gene products control a broad set of disease resistance responses whose induction is often sufficient to stop further pathogen growth/spread (Agrios, 1988).

Studied R genes usually confer specificity for particular strains of a pathogen species (those that express the recognized effector). As first noted by Harold Flor in his mid-20th century formulation of the gene-for-gene relationship, a plant $R$ gene has specificity for a pathogen avirulence gene (Avr gene). Avirulence genes 
are now known to encode effectors. The pathogen $A v r$ gene must have matched specificity with the $R$ gene for that $R$ gene to confer resistance, suggesting a receptor/ligand interaction for $A v r$ and $R$ genes. Alternatively, an effector can modify its host cellular target (or a molecular decoy of that target), and the $R$ gene product (NLR protein) activates defenses when it detects the modified form of the target or decoy. Resistance genes ( $R$-Genes) are genes in plant genomes that convey plant disease resistance against pathogens by producing $\mathrm{R}$ proteins (Ellis et al., 2000). These are genes in plant genomes that convey plant disease resistance against pathogens by producing $\mathrm{R}$ proteins. Many plant-pathogen interactions are determined by the presence of resistance $(R)$ genes/alleles that enable plants to recognize pathogens and activate inducible defenses. Encode proteins that recognize Avr-genedependent ligands, Activate signaling cascade(s) that coordinate the initial plant defense responses to impair pathogen ingress (Ellis and Jones, 1998).

\section{Classes of $\boldsymbol{R}$ genes}

There are five classes of $R$ genes based on the structural characteristics of their protein product. The first class codes for serine/threonine kinase with the Pto gene as the only known member. It confers resistance to bacterial speck in tomato and encodes a serine/threonine protein kinase with no leucine rich repeat (LRR). The second class includes $R$ genes coding for receptor-like protein with a transmembrane domain and an extracellular LRR domain. Members of this class include $c f$ gene product mainly found in Solanaceous species and specific for leaf mold resistance and HSI specific for nematode resistance. The third class of $R$ genes codes for receptor-like kinase with an extracellular LRR, a membrane-spanning region and an intracellular protein kinase domain. This class is exemplified by Xa21, Xa26 and Pi-d2 in rice. However, $\mathrm{Xa} 21$ is now classified as a PRR since it is known that it recognizes the PAMP Ax21 (or its eliciting epitope axYs22), a type I secreted sulphated protein that is conserved across Xanthomonas species and a few related species. It has been shown that a tyrosine-sulphated 17amino acid synthetic peptide corresponding to the $\mathrm{N}$ terminus of Ax21 is fully active in eliciting Xa21mediated resistance. Ax21 has been shown to bind directly to Xa21 and the tyrosine sulphation is required for recognition by Xa21 (Shen et al., 2002).

The fourth class includes the vast majority of $R$ genes known as the nucleotide binding-site leucine rich repeat (NBS-LRR) resistance genes (Meyers et al., 1999; van der Biezen and Jones 1998). NBS-LRR genes are abundant in plants. For instance it estimated that at least 200 different NBS-LRR genes exist in Arabidopsis, representing $1 \%$ of its genome (Ellis et al., 2000). The last class includes all $\mathrm{R}$ genes which cannot be fitted in any of the above four classes due to their different structure (van der Biezen and Jones, 1998). An example is the powdery mildew resistance gene, RPW8, in Arabidopsis. It codes for a small protein with only an amino-terminal transmembrane domain and a coiled coil domain and it confers an unusually broad spectrum of resistance (Meyers et al., 1999).

Classes 1 and $4 \mathrm{R}$ genes are predominantly or completely intracellular and therefore recognize intracellular ligands. On the other hand, classes 2 and 3 $R$ genes may recognize extracellular ligands due to the presence of transmembrane domains. It is known that for $\mathrm{R}$ proteins in classes 2, 3 and 4 the LRR domain is responsible for specific recognition of pathogen-derived ligand. Moreover, mutational analysis has revealed that non-LRR and LRR-containing $\mathrm{R}$ proteins may use common pathways. For instance, Pto resistance is dependent on the NB-LRR protein PRF and Rpw8 requires Eds1 (an $R$ gene signaling component used by several NB-LRR R proteins) to function. However, other studies of the Arabidopsis NB-LRR $R$ gene RPS5 have indicated that LRRs are also involved in downstream signaling.

\section{Resistance can be conveyed through a number of mechanisms including (van der Plank, 1968):}

1. The $\mathrm{R}$ protein interacts directly with an $A v r$ gene (Avirulence gene) product of a pathogen (Gene-for-Gene relationship).

2. The $\mathrm{R}$ protein guards another protein that detects degradation by an Avr gene (Guard hypothesis).

3. The $\mathrm{R}$ protein may detect a PathogenAssociated Molecular Pattern or PAMP (alternatively called MAMP for microbeassociated molecular pattern).

4. The $\mathrm{R}$ protein encodes enzyme that degrades a toxin produced by a pathogen.

Once the $\mathrm{R}$ protein has detected the presence of a pathogen, the plant can mount a defence against the pathogen. Because $R$ genes confer resistance against 
specific pathogens, it is possible to transfer an $\mathrm{R}$ gene from one plant to another and make a plant resistant to a particular pathogen. Although plant disease resistance is a complex phenomenon involving a multitude of genes and several interconnected signaling pathways, a reasonably clear picture has begun to emerge, based largely on research conducted in Arabidopsis and supported by work in various other species (for recent reviews. Resistance to fungal and bacterial pathogens often involves the induction of the hypersensitive response (HR) and the development of systemic acquired resistance (SAR) via the salicylic acid (SA) signaling pathway (Robinson, 1971). HR involves localized expression of pathogenesisrelated (PR) proteins and causes localized host cell death and callose deposition at the site of infection, thereby restricting fungal growth and limiting the spread of disease. SAR is characterized by systemic broadspectrum resistance to virulent pathogens and involves the transcriptional activation of $P R$ genes at sites distant from the site of initial infection.

\section{Gene-for-gene relationship (Flor, 1971)}

The gene-for-gene relationship was discovered by the late Harol Henry Flor who was working with rust (Melampsora lini) of flax (Linum usitatissimum). Flor showed that the inheritance of both resistance in the host and parasite ability to cause disease is controlled by pairs of matching genes. One is a plant gene called the resistance $(R)$ gene. The other is a parasite gene called the avirulence $(A v r)$ gene. Plants producing a specific $\mathrm{R}$ gene product are resistant towards a pathogen that produces the corresponding Avr gene product. Gene-forgene relationships are a widespread and very important aspect of plant disease resistance (Allard, 1960).

\section{Guard hypothesis}

In only some cases is there direct interaction between the $R$ gene product and the $A v r$ gene product. For example, both FLS2 and XA21 interact with the microbial peptides. In contrast, for the NBS-LRR class of $R$ genes, direct interaction has not been shown for most of the $R / A v r$ pairs. This lack of evidence for a direct interaction led to the formation of the guard hypothesis for the NBS-LRR class of $R$ genes. This model proposes that the $\mathrm{R}$ proteins interact, or guard, a protein known as the guardee which is the target of the Avr protein. When it detects interference with the guardee protein, it activates resistance.

Several experiments support this hypothesis, e.g. the
Rpml gene in Arabidopsis thaliana is able to respond to two completely unrelated avirulence factors from Pseudomona syringae. The guardee protein is RIN4, which is hyperphosphorylated by the Avr proteins. Another high profile study that supports the guard hypothesis shows that the RPS5 pair uses PBS1, a protein kinase as a guardee against AvrPphB.

Yeast two-hybrid studies of the tomato Pto/Prf/AvrPto interaction showed that the avirulence protein, AvrPto, interacted directly with Pto despite Pto not having an LRR. This makes Pto the guardee protein, which is protected by the NBS-LRR protein Prf. However, Pto is a resistance gene alone, which is an argument against the guard hypothesis (Sidhu, 1975).

\section{Pathogen-associated molecular patterns or PAMPs}

These are molecules associated with groups of pathogens, that are recognized by cells of the innate immune system. These molecules can be referred to as small molecular motifs conserved within a class of microbes. They are recognized by Toll-like receptors (TLRs) and other pattern recognition receptors (PRRs) in both plants and animals. A vast array of different types of molecules can serve as PAMPs, including glycans and glycoconjugates. PAMPs activate innate immune responses, protecting the host from infection, by identifying some conserved nonself molecules.

Bacterial lipopolysaccharides (LPSs), endotoxins found on the cell membranes of bacteria, are considered to be the prototypical class of PAMPs. LPSs are specifically recognised by TLR4, a recognition receptor of the innate immune system. Other PAMPs include bacterial flagellin (recognized by TLR5), lipoteichoic acid from gram-positive bacteria, peptidoglycan, and nucleic acid variants normally associated with viruses, such as double-stranded RNA (dsRNA), recognized by TLR3 or unmethylated $\mathrm{CpG}$ motifs, recognized by TLR9 (Gururani et al., 2012). Although the term "PAMP" is relatively new, the concept that molecules derived from microbes must be detected by receptors from multicellular organisms has been held for many decades, and references to an "endotoxin receptor" are found in much of the older literature.

\section{Conclusion}

The ability of populations of viral, bacterial and fungal pathogens to shift to virulent biotypes is well known in 
field situations. Thus the ability of plant species to survive over evolutionary time might depend on their ability to generate useful diversity at resistance gene loci. Our understanding of the nature, genome organization and evolution of $\mathrm{R}$ gene loci has increased immensely in recent years (Brown, 2002). Common conclusions in the evolution of $\mathrm{R}$ genes and gene families are emerging on the basis of sequence analysis of cloned $\mathrm{R}$ genes. The ancient nature of NBS-LRR sequences, their separation into distinct lineages and more recent diversification helps to explain the observed sequence diversity and structural features of this gene family. At a genomic level, extensive gene clusters are a striking property of most $\mathrm{R}$ genes that is probably related to a balance between creating new specificities and conserving old ones. The well-known mechanisms for generating diversity of $\mathrm{R}$ genes are duplication and recombination. Then a gene family is formed by chromosomal duplication and a subsequent divergence of progenitor sequences. This process creates new loci, changes the number of family members, and provides homologous sequences for recombination and unequal crossing-over events.

\section{Conflict of interest statement}

Authors declare that they have no conflict of interest.

\section{References}

Agrios, G. N., 1988. Plant Pathology. $3^{\text {rd }}$ Edn. Academic Publ., London.

Allard, R. W., 1960. Principles of Plant Breeding. John Wiley and Sons, Inc., New York.

Brown, J. K. M., 2002. Yield penalties of disease resistance in crops. Curr. Opin. Biosci. 5, 339-344.
Ellis, J., Dodds, P., Pryor, T., 2000. Structure, function and evolution of plant disease resistance genes. Curr. Opin. Plant Biol. 3, 278-284.

Ellis, J., Jones, D., 1998. Structure and function of proteins controlling strain-specific pathogen resistance in plants. Curr. Opin. Plant Biol. 1, 288293.

Flor, H. H., 1971. Current status of the gene-for-gene concept. Annu. Rev. Phytopathol. 9, 278-296.

Gururani, M. A., Venkatesh, J., Upadhyaya, C. P., Nookaraju, A., Pandey, S. K., Park, S. W., 2012. Plant disease resistance genes: Current status and future directions. Physiol. Mol. Plant Pathol. 78, 5165.

Meyers, B.C., Dickerman, A.W., Michelmore, R.W., Sivaramakrishnan, S., Sobral, B. W., Young, N.D., 1999. Plant disease resistance genes encode members of an ancient and diverse protein family within the nucleotide-binding superfamily. Plant J. 20, 317-332.

Robinson, R. A., 1971. Vertical resistance. Rev. Plant Pathol. 50, 233-239.

Shen, Y., Sharma, P., Silva, F. G., Ronald, P., 2002. The Xanthomonas oryzae pv. lozengeoryzae $\operatorname{rax} P$ and $\operatorname{rax} Q$ genes encode an ATP sulphurylase and adenosine-50 -phosphosulphate kinase that are required for AvrXa21 avirulence activity. Mol. Microbiol. 44, 37-48.

Sidhu, G., 1975. Gene-for-gene relationships in plant parasitic systems. Sci. Progress (Oxford) 62, 467485.

van der Biezen, E. A., Jones, J. D., 1998. Plant diseaseresistance proteins and the gene-for-gene concept. Trends Biochem. Sci. 23, 454-456.

van der Plank, J. E., 1968. Disease Resistance in Plants. Academic Press, New York and London.

\section{How to cite this article:}

Jatwa, T.K., Sharma, M., Malav, A. K., 2017. R gene and its role in disease managements in plants. Int. J. Curr. Res. Biosci. Plant Biol. 4(5), 61-64. doi: https://doi.org/10.20546/ijcrbp.2017.405.008 